\title{
Evaluation of Real-World Experience with Tofacitinib Compared with Adalimumab, Etanercept, and Abatacept in RA Patients with 1 Previous Biologic DMARD: Data from a U.S. Administrative Claims Database
}

\author{
James Harnett, PharmD, MS; Robert Gerber, PharmD; David Gruben, PhD; \\ Andrew S. Koenig, DO; and Connie Chen, PharmD
}

\begin{abstract}
BACKGROUND: Real-world data comparing tofacitinib with biologic disease-modifying antirheumatic drugs (bDMARDs) are limited.
\end{abstract}

OBJECTIVE: To compare characteristics, treatment patterns, and costs of patients with rheumatoid arthritis $(\mathrm{RA})$ receiving tofacitinib versus the most common bDMARDs (adalimumab [ADA], etanercept [ETN], and abatacept [ABA]) following a single bDMARD in a U.S. administrative claims database.

METHODS: This study was a retrospective cohort analysis of patients aged $\geq 18$ years with an RA diagnosis (ICD-9-CM codes $714.0 x-714.4 x ; 714.81$ ) and 1 previous bDMARD filling $\geq 1$ tofacitinib or bDMARD claim in the Truven MarketScan Commercial and Medicare Supplemental claims databases (November 1, 2012-0ctober 31, 2014). Monotherapy was defined as absence of conventional synthetic DMARDs within 90 days post-index. Persistence was evaluated using a 60-day gap. Adherence was assessed using proportion of days covered (PDC). RA-related total, pharmacy, and medical costs were evaluated in the 12-month pre- and post-index periods. Treatment patterns and costs were adjusted using linear models including a common set of clinically relevant variables of interest (e.g., previous RA treatments), which were assessed separately using t-tests and chi-squared tests.

RESULTS: 0verall, 392 patients initiated tofacitinib; 178 patients initiated ADA; 118 patients initiated ETN; and 191 patients initiated ABA. Tofacitinib patients were older versus ADA patients $(P=0.0153)$ and had a lower proportion of Medicare supplemental patients versus ABA patients $(P=0.0095)$. Twelve-month pre-index bDMARD use was greater in tofacitinib patients (77.6\%) versus bDMARD cohorts (47.6\%-59.6\%). Tofacitinib patients had greater 12-month pre-index RA-related total costs versus bDMARD cohorts (all $P<0.0001$ ) and greatest index use of monotherapy $(P=0.0080$ vs. ABA). A similar (all $P>0.10$ ) proportion of patients were persistent with tofacitinib (42.6\%) versus ADA (37.6\%), ETN (42.4\%), and ABA (43.5\%). Mean PDC was 0.55 for tofacitinib versus 0.57 (ADA), 0.59 (ETN), and 0.44 ( $A B A ; P=0.0003)$. Adjusted analyses generated similar findings to the unadjusted treatment patterns. Tofacitinib had lower adjusted 12-month post-index mean RA-related total costs $(\$ 23,568)$ versus ADA $(\$ 29,278$; $P<0.0001)$, ETN $(\$ 26,885 ; P=0.0248)$, and ABA $(\$ 30,477 ; P<0.0001)$.

CONCLUSIONS: In this study, tofacitinib was more commonly used as monotherapy and yielded at least comparable persistence and adherence with lower adjusted mean RA-related total costs versus ADA, ETN, and $A B A$. Further analysis is warranted given the greater 12-month pre-index bDMARD use and RA-related costs for tofacitinib versus bDMARDs.

J Manag Care Spec Pharm. 2016;22(12):1457-71

Copyright $\odot 2016$, Academy of Managed Care Pharmacy. All rights reserved.

\section{What is already known about this subject}

Tofacitinib is an oral Janus kinase inhibitor for the treatment of rheumatoid arthritis (RA).

A network meta-analysis of randomized controlled trial data has demonstrated that tofacitinib has similar efficacy to biologic disease-modifying antirheumatic drugs (bDMARDs) in improving the signs and symptoms of RA in patients with an inadequate response to tumor necrosis factor inhibitors.

\section{What this study adds}

Although most patients received a conventional synthetic DMARD (csDMARD) in the 12-month pre-index period, a substantial proportion started a bDMARD, or more commonly, tofacitinib monotherapy; those receiving tofacitinib monotherapy had the lowest proportion initiating csDMARDs in the 12-month follow-up period (i.e., stayed on monotherapy) versus those receiving bDMARDs. Tofacitinib patients were more likely to have received a bDMARD in the 12-month pre-index period and had at least comparable persistence and adherence versus patients receiving bDMARDs. Total RA-related costs in the 12-month post-index period adjusted for demographics and prior enrollment history, clinical characteristics, prior RA-related treatments, use of a monotherapy regimen, and pre-index RA-related costs were significantly lower for patients receiving tofacitinib versus patients receiving bDMARDs.

$\mathrm{R}$ heumatoid arthritis (RA) is a chronic, progressive, and disabling autoimmune disease affecting 1.5 million people in the United States. ${ }^{1}$ The economic burden of RA is substantial. Data from U.S. administrative claims databases covering privately insured and Medicare/Medicaid beneficiaries reported RA costs of $\$ 19.3$ billion annually, when intangible costs were excluded, and $\$ 39.2$ billion when these costs were considered. ${ }^{2}$ U.S. Medical Expenditure Panel Survey data suggest an annual cost of $\$ 22.3$ billion. $^{3}$

The current goal of RA treatment is to achieve low disease activity or remission. ${ }^{4,5}$ The American Rheumatism Association early response criteria included a "remission" definition; ${ }^{6}$ however, at that time, therapies were rarely able 
to achieve such low activity levels. In contemporary practice, there is a greater ability to limit or eliminate clinical signs and symptoms, as exemplified in the American College of Rheumatology/European League Against Rheumatism (ACR/EULAR) Boolean response criteria. ${ }^{7}$ The mainstay of initial disease-modifying antirheumatic drug (DMARD) therapy is methotrexate (MTX), which reduces signs and symptoms of RA and slows progression of joint damage. However, intensive MTX monotherapy achieves remission in only one third of patients. ${ }^{8}$ Administering MTX with biologic DMARDs (bDMARDs) can optimize efficacy, as measured by clinical, functional, patient-reported, and radiologic outcomes..$^{9-13}$

The introduction of bDMARDs has improved the lives of many RA patients; however, only one third achieve clinical remission..$^{14}$ bDMARDs include the following: tumor necrosis factor inhibitors (TNFi): adalimumab (ADA), etanercept (ETN), infliximab (IFX), certolizumab pegol (CZP), and golimumab (GOL); an interleukin (IL)-6 receptor inhibitor: tocilizumab (TCZ); an IL-1 receptor inhibitor: anakinra (ANK); a B-cell depleting anti-CD20 monoclonal antibody: rituximab (RTX); and a co-stimulation blocker of cytotoxic T lymphocyte antigen 4: abatacept (ABA). TNFi can be ineffective in some patients because of individual variations in pathophysiological response or differences in the dominant cytokine mediating the disease process. ${ }^{15}$ Patients may develop secondary loss of efficacy following auto-antibody production ${ }^{16-18}$ necessitating concomitant conventional synthetic DMARDs (csDMARDs) and dose escalation, most commonly with IFX and ADA. ${ }^{19-21}$ bDMARD use may also be limited by intravenous or subcutaneous administration, which is inconvenient and associated with infusion/ injection-site reactions, ${ }^{16-18}$ substantial costs,${ }^{22}$ and compliance issues if doses are reduced because of fear of adverse effects or actual intolerance. ${ }^{23}$

Tofacitinib is an oral Janus kinase (JAK) inhibitor for the treatment of RA. Tofacitinib $5 \mathrm{mg}$ twice daily (BID) and, more recently, once-daily dosing are approved for use in the United States in adults with moderately to severely active RA and an inadequate response/intolerance to MTX and can be administered alone or with csDMARDs. ${ }^{24}$ ACR published updated 2015 treatment guidelines recommending tofacitinib (and bDMARDs) for RA-established patients who have inadequate response to csDMARDs. ${ }^{5}$ In current practice, and supported by U.S. payer access, TNFi (particularly ADA and ETN) are commonly used after csDMARDs. For patients with inadequate responses to their first bDMARDs, ACR recommends non-TNFi bDMARDs before TNFi and tofacitinib; however, this is supported by very low-level evidence.

In this study, U.S. administrative claims data were used to compare patient characteristics, treatment patterns, and costs in RA patients receiving tofacitinib or common bDMARDs following 1 previous bDMARD.

\section{Methods}

\section{Study Design}

This study was a retrospective cohort analysis of patients aged $\geq 18$ years (at index) with RA (International Classification of Diseases, Ninth Revision, Clinical Modification [ICD-9-CM] codes $714.0 \mathrm{x}-714.4 \mathrm{x}$ and 714.81$)$ and $\geq 1$ claim for tofacitinib (selected first) or a bDMARD between November 1, 2012, and October 31, 2014, in the Truven MarketScan Commercial and Medicare Supplemental databases. Intravenous and subcutaneous bDMARDs included TNFi (ADA, ETN, IFX, CZP, and GOL) and non-TNFi (ABA, RTX, TCZ, and ANK) bDMARDs. The index event was the first tofacitinib or bDMARD use at which patients had continuous enrollment $\geq 12$ months before and $\geq 12$ months after index treatment and fulfilled other selection criteria. Primary baseline period was 12 months preindex; however, a variable-length pre-index, using the earliest continuous enrollment available through index, and any preindex (i.e., all available claims) periods, were explored.

Excluded patients had another inflammatory condition for which bDMARDs are used (Crohn's disease [555.xx], ulcerative colitis [556.xx], ankylosing spondylitis [720.0x], psoriasis [696.1x], and psoriatic arthritis [696.0x]) in the 12-month preindex period or at index and previous use of index medication or filled $>1$ bDMARD or a bDMARD with tofacitinib at index.

This analysis focused on patients with 1 previous nonindex bDMARD at any time before index, since a significant proportion of patients received bDMARDs before the 12-month preindex period. An imbalance in the number of patients with $\geq 2$ previous bDMARDs was also observed particularly for ADA and ETN, which were most common in bDMARD-naive patients.

\section{Comparisons}

Among patients who received 1 bDMARD pre-index, those who initiated treatment with tofacitinib were compared with those initiating ADA, ETN, or ABA (the most common bDMARDs used in these patients).

\section{Assessments}

Patient Characteristics. Patient characteristics included baseline demographics, 12-month pre-index use of DMARDs and other RA-related medications, rheumatologist visits, QuanCharlson Comorbidity Index $(\mathrm{CCI})^{25}$ and claims-based index of RA severity (CIRAS) scores, ${ }^{26}$ use of bDMARDs any time before index, and number of days from earliest diagnosis in the variable-length baseline period to index.

Treatment Patterns. Monotherapy was defined as the absence of select csDMARDs (MTX, sulfasalazine, hydrochloroquine, or leflunomide) within 90 days post-index. For monotherapy patients, addition of select csDMARDs in the 12-month postindex period was evaluated. 
Treatment persistence was defined as continuing index medication without a 60-day gap after the prescription days supply had run out through to the end of the 12-month postindex period. ${ }^{27-29}$ Early switch was defined as the initiation of tofacitinib or a nonindex bDMARD (including the bDMARDs previously listed) during or before a 60-day gap in index treatment. Delayed switch was defined as the initiation of tofacitinib or a nonindex bDMARD after a gap in index therapy $\geq 60$ days.

Proportion of days covered (PDC) was derived by calculating the number of days covered by the prescription fills during the denominator period. ${ }^{30}$ The numerator consisted of days supply covered by arrays for each fill during the denominator period and adjusted for a maximum 15-day overlap in days supply. The denominator was the time from index medication fill/administration to the end of the 12-month post-index period. PDC was capped at 1.

In the study cohort, $81 \%$ of index medication prescriptions had a 28-day supply, and 13\% had an 84-day supply across ADA, ETN, and subcutaneous ABA groups. For tofacitinib, $87 \%$ had a 30 -day supply, and $11 \%$ had a 90 -day supply. Total days supply for tofacitinib, ADA, ETN, and subcutaneous ABA was the days supply rounded to the nearest 28-day period. Rounding was implemented to address likely inaccurate days supply based on the analysis of index medication prescription days supply and for consistency with dosing recommendations, as well as to standardize across monthly supplies (i.e., 28 days [bDMARDs] vs. 30 days [tofacitinib]). ABA infusion used a 28-day supply.

Weekly dose was determined for index claims during the follow-up period and calculated by dividing the total dose per prescription by the total days supply multiplied by 7 days. Consistent with previous publications, ${ }^{31,32}$ dose escalation was defined as any increase in an average weekly dose to $\geq 40 \mathrm{mg}$ per week for ADA and $\geq 100 \mathrm{mg}$ per week for ETN. An increase of at least double the recommended dose for tofacitinib (i.e., $\geq 20 \mathrm{mg}$ per day) and subcutaneous ABA (i.e., $\geq 250 \mathrm{mg}$ per week) was considered a dose escalation. Dose escalation for intravenous ABA occurred if the last dose was $\geq 100 \mathrm{mg}$ greater than the first dose. ${ }^{32}$

Costs. All-cause and RA-related health care resource use and costs were evaluated during the 12-month pre- and post-index periods. RA-related visits included those with ICD-9-CM code 714.xx in any position on the claim and any bDMARD administration-related visit (using associated Healthcare Common Procedure Coding System codes regardless of RA diagnosis, but excluding an osteoporosis diagnosis [ICD-9-CM code 733.0]) if observed in the absence of an RA diagnosis (i.e., not be classified as RA related). RA-related prescriptions included csDMARDs and bDMARDs, COX-2 inhibitors, nonselective nonsteroidal anti-inflammatory drugs, opioids, and glucocorticoids. Costs included patient and health plan-paid amounts.
Costs were adjusted using the annual medical care component of the Consumer Price Index to reflect inflation between 2011 (earliest pre-index period) and 2013.

\section{Statistical Analyses}

Simple statistical analyses were performed using pairwise t-statistics (continuous variables) or chi-squared statistics (binary variables) for all variables considered clinically relevant in comparing each bDMARD medication at index versus tofacitinib. These variables included demography, enrollment history, clinical characteristics, previous RA-related treatments, and use of a monotherapy regimen (Appendix, available in online article). The behavior of the t-test was compared with a nonparametric test (i.e., Wilcoxon), particularly for data that were likely to be skewed. The t-test was robust and chosen for simplicity and comparability with the adjusted analyses.

Adjusted analyses were performed using generalized linear models. Each model included clinically relevant variables. For cost-related dependent variables, the model also included the corresponding 12-month pre-index cost. Binary variables were analyzed with a logit link. Cost variables were analyzed as gamma-distributed data with a log link. Other variables were treated as normally distributed with an inverse link. Pairwise t-statistics and chi-square statistics were also applied to the dependent variables as an initial check, comparing the bDMARDs at index versus tofacitinib.

For independent variables, $P$ values $\leq 0.05$ were noted for the relative importance of that independent variable. For each variable, when comparing index medication, $P$ values were significant after applying the multiple comparison procedure due to Hochberg, versus tofacitinib, using the 0.05 significance level, unless otherwise noted. ${ }^{33}$

For adjusted and unadjusted analyses, missing values were ignored. All $P$ values were 2-sided.

\section{Results}

\section{Patients}

From November 2012 to October 2014, 1,252 patients initiated tofacitinib or bDMARDs and met the initial study selection criteria (Figure 1). Overall, 392 (31.3\%) patients received index treatment with tofacitinib, and 178 (14.2\%), 118 (9.4\%), and 191 (15.3\%) patients initiated with ADA, ETN, and ABA, respectively. The remaining $373(29.8 \%)$ patients were treated with CZP ( $n=57 ; 4.6 \%)$, IFX ( $n=59 ; 4.7 \%)$, RTX ( $n=88 ; 7.0 \%)$, TCZ ( $n=95 ; 7.6 \%)$, GOL $(n=70 ; 5.6 \%)$, and ANK $(n=4 ; 0.3 \%)$, and are not reported on further.

Although patient demographics and clinical characteristics were similar between patients receiving tofacitinib and the 3 most common bDMARDs, there were differences, such as tofacitinib patients were older than ADA patients $(P=0.0153)$ and had a lower proportion of Medicare supplemental patients than ABA patients $(P=0.0095)$. A greater proportion of 
Evaluation of Real-World Experience with Tofacitinib Compared with Adalimumab, Etanercept, and Abatacept in RA Patients with 1 Previous Biologic DMARD: Data from a U.S. Administrative Claims Database

\section{FIGURE 1 Cohort Selection}

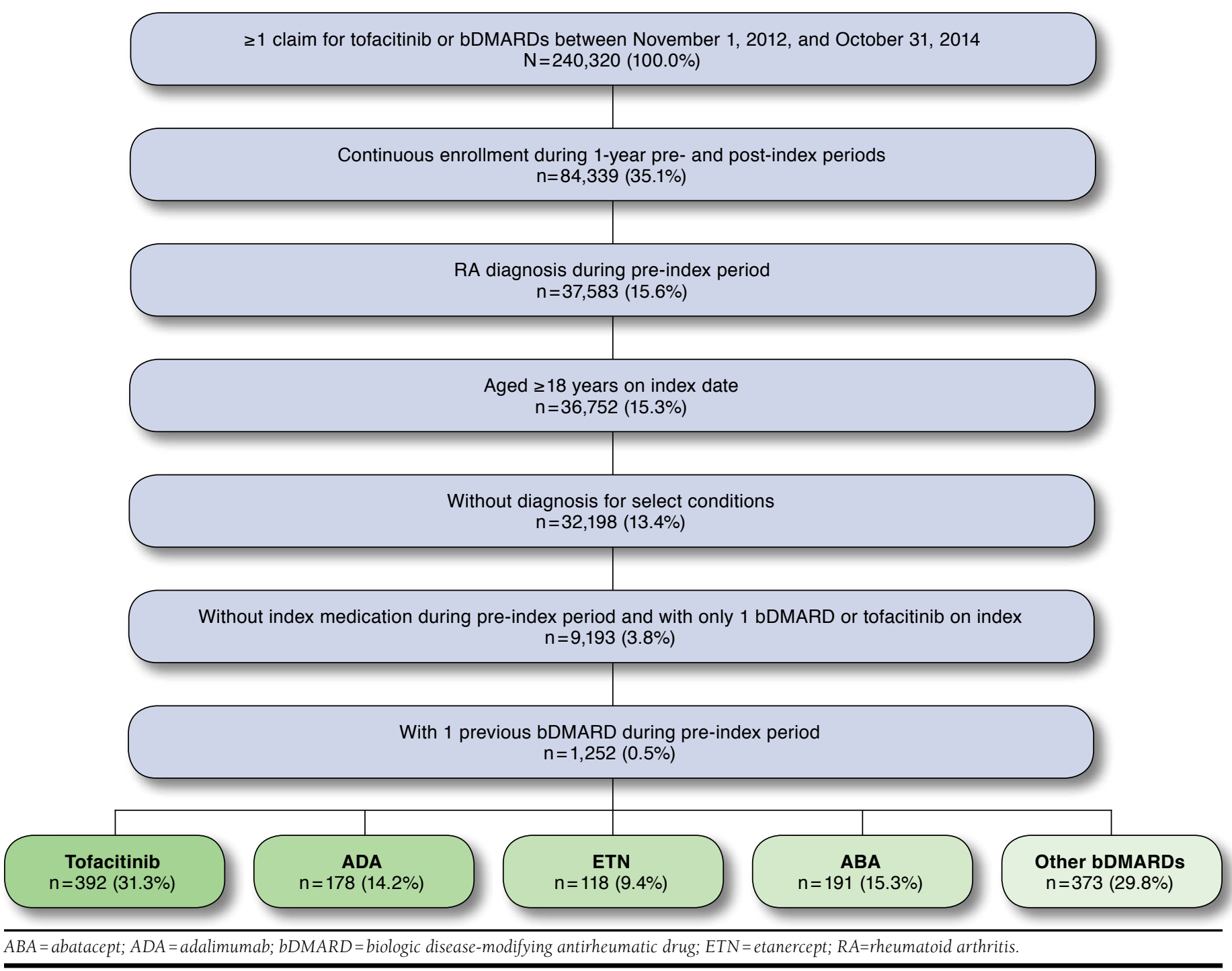

tofacitinib patients received bDMARDs in the 12-month preindex period versus those receiving ADA, ETN, and ABA (all $P<0.0001$; Table 1).

TNFi were the most common bDMARDs at any time preindex across all groups. A greater proportion of ADA, ETN, and ABA patients received TNFi at any time pre-index versus tofacitinib patients (all $P<0.05$ ). A greater proportion of tofacitinib patients received a non-TNFi bDMARD at any time pre-index versus ADA, ETN, and ABA patients (all $P<0.05$ ). Most patients receiving tofacitinib, ADA, ETN, and ABA received treatment with csDMARDs in the 12-month preindex period. MTX was the most common csDMARD in the 12-month pre-index period (Table 1).

\section{Twelve-Month Post-index Treatment Patterns}

Overall, 53.1\% (208/392) of patients received tofacitinib as monotherapy versus 48.3\% (86/178), 48.3\% (57/118), and 41.4\% (79/191; $P=0.0080)$ of patients receiving ADA, ETN, and ABA, respectively. The remaining patients received combination therapy with csDMARDs, most commonly MTX (70.2\%). Of patients who initiated tofacitinib as monotherapy, $24.0 \%$ (50/208) received a csDMARD in the 12-month post-index period versus 34.9\% (30/86), 29.8\% (17/57), and 39.2\% (31/79; $P=0.0106)$ of patients initiating ADA, ETN, and ABA monotherapy regimens, respectively.

A similar proportion of patients initiating tofacitinib (42.6\%; 167/392), ADA (37.6\%; 67/178), ETN (42.4\%; 50/118), and 
Evaluation of Real-World Experience with Tofacitinib Compared with Adalimumab, Etanercept, and Abatacept in RA Patients with 1 Previous Biologic DMARD: Data from a U.S. Administrative Claims Database

TABLE 1 Patient Demographics and Baseline Characteristics in the 12-Month Pre-index Period (Unless Noted Otherwise)

\begin{tabular}{|c|c|c|c|c|c|c|c|}
\hline & $\begin{array}{l}\text { Tofacitinib } \\
\mathbf{n}=392\end{array}$ & $\begin{array}{c}\text { ADA } \\
\mathrm{n}=178\end{array}$ & $\begin{array}{c}P \text { Value } \\
\text { ADA vs. } \\
\text { Tofacitinib }\end{array}$ & $\begin{array}{c}\text { ETN } \\
\mathrm{n}=118\end{array}$ & $\begin{array}{c}P \text { Value } \\
\text { ETN vs. } \\
\text { Tofacitinib }\end{array}$ & $\begin{array}{c}\text { ABA } \\
n=191\end{array}$ & $\begin{array}{l}\text { P Value } \\
\text { ABA vs. } \\
\text { Tofacitinib }\end{array}$ \\
\hline Age in years, mean [SD] & $\begin{array}{ll}54.6 & {[11.7]} \\
\end{array}$ & $\begin{array}{ll}51.5 & {[15.2]} \\
\end{array}$ & $0.0153^{a}$ & $\begin{array}{ll}52.8 & {[13.7]} \\
\end{array}$ & 0.1814 & $\begin{array}{ll}55.9 & {[12.6]} \\
\end{array}$ & 0.2179 \\
\hline Female, n (\%) & $328 \quad(83.7)$ & $156 \quad(87.6)$ & 0.2201 & $100 \quad(84.8)$ & 0.7810 & $160 \quad(83.8)$ & 0.9765 \\
\hline Geographic region, n (\%) & & & 0.3270 & & 0.2068 & & 0.5078 \\
\hline South & $168 \quad(42.9)$ & $62 \quad(34.8)$ & & $45 \quad(38.1)$ & & $72 \quad(37.7)$ & \\
\hline North Central & $91 \quad(23.2)$ & $50 \quad(28.1)$ & & $37 \quad(31.4)$ & & $56 \quad(29.3)$ & \\
\hline West & $66 \quad(16.8)$ & $38 \quad(21.4)$ & & $22 \quad(18.6)$ & & $31 \quad(16.2)$ & \\
\hline Northeast & $60 \quad(15.3)$ & $25 \quad(14.0)$ & & $14 \quad(11.9)$ & & $27 \quad(14.1)$ & \\
\hline Unknown & $7 \quad(1.8)$ & $3 \quad(1.7)$ & & - & & $5 \quad(2.6)$ & \\
\hline Data provider, $\mathrm{n}(\%)$ & & & 0.0857 & & 0.3134 & & 0.1139 \\
\hline Employer & $284 \quad(72.5)$ & $141 \quad(79.2)$ & & $91 \quad(77.1)$ & & $150 \quad(78.5)$ & \\
\hline Health plan & $108 \quad(27.6)$ & $37 \quad(20.8)$ & & $27 \quad(22.9)$ & & $41 \quad(21.5)$ & \\
\hline Insurance, n (\%) & & & 0.2911 & & 0.9019 & & $0.0095^{a}$ \\
\hline Commercial & $327 \quad(83.4)$ & 142 (79.8) & & 99 (83.9) & & $142 \quad(74.4)$ & \\
\hline Medicare supplemental & $65 \quad(16.6)$ & $36 \quad(20.2)$ & & $19 \quad(16.1)$ & & $49 \quad(25.7)$ & \\
\hline $\begin{array}{l}\text { Time in days between earliest RA diagnosis } \\
\text { observed to index date, mean [SD] }\end{array}$ & $930.9[423.4]$ & 915.7 [389.6] & 0.6840 & $865.9[340.7]$ & 0.0883 & $967.1[360.6]$ & 0.2834 \\
\hline CCI score, mean [SD] & $1.5 \quad[1.0]$ & {$[1.1]$} & 0.2347 & {$[1.2]$} & 0.9548 & {$[1.0]$} & 0.6138 \\
\hline CIRAS score, mean [SD] & $4.6 \quad[1.4]$ & {$[1.6]$} & 0.0517 & {$[1.5]$} & 0.0788 & $4.5 \quad[1.4]$ & 0.5349 \\
\hline Rheumatologist visits, mean [SD] & $5.0 \quad[3.2]$ & $4.2 \quad[2.7]$ & $0.0103^{\mathrm{a}}$ & $4.3 \quad[3.1]$ & 0.1062 & $4.8 \quad[3.6]$ & 0.5828 \\
\hline bDMARD use, n (\%) & $304 \quad(77.6)$ & $106 \quad(59.6)$ & $<0.0001^{\mathrm{a}}$ & $58 \quad(49.2)$ & $<0.0001^{\mathrm{a}}$ & $91 \quad(47.6)$ & $<0.0001^{\mathrm{a}}$ \\
\hline bDMARD use (any time pre-index), n (\%) & $392(100.0)$ & $178(100.0)$ & & $118(100.0)$ & & $191(100.0)$ & \\
\hline TNFi & $288 \quad(73.5)$ & $163(91.6)$ & $<0.0001^{\mathrm{a}}$ & $101 \quad(85.6)$ & $0.0066^{\mathrm{a}}$ & $177 \quad(92.7)$ & $<0.0001^{\mathrm{a}}$ \\
\hline $\mathrm{ADA}$ & $100 \quad(25.5)$ & N/A & N/A & $76 \quad(64.4)$ & $<0.0001^{\mathrm{a}}$ & $62 \quad(32.5)$ & 0.0787 \\
\hline $\mathrm{CZP}$ & $(4.6)$ & $(0.6)$ & $0.013^{\mathrm{a}}$ & $(4.2)$ & 0.8707 & $(4.7)$ & 0.9483 \\
\hline ETN & $109 \quad(27.8)$ & $127 \quad(71.4)$ & $<0.0001^{\mathrm{a}}$ & N/A & N/A & $69 \quad(36.1)$ & $0.0406^{\mathrm{a}}$ \\
\hline IFX & $37 \quad(9.4)$ & $25 \quad(14.0)$ & 0.1017 & $14 \quad(11.9)$ & 0.4413 & $33 \quad(17.3)$ & $0.0063^{a}$ \\
\hline GOL & $24 \quad(6.1)$ & $10 \quad(5.6)$ & 0.8137 & $(5.1)$ & 0.6745 & $(2.1)$ & 0.0328 \\
\hline Non-TNFi bDMARD & $104 \quad(26.5)$ & $15 \quad(8.4)$ & $<0.0001^{\mathrm{a}}$ & $17 \quad(14.4)$ & $0.0066^{\mathrm{a}}$ & $(7.3)$ & $<0.0001^{\mathrm{a}}$ \\
\hline RTX & $21 \quad(5.4)$ & $4 \quad(2.3)$ & 0.0929 & $(5.1)$ & 0.9078 & $10 \quad(5.2)$ & 0.9511 \\
\hline $\mathrm{TCZ}$ & $26 \quad(6.6)$ & 0 & $0.0004^{a}$ & 0 & $0.0041^{\mathrm{a}}$ & $(1.6)$ & $0.0083^{a}$ \\
\hline $\mathrm{ABA}$ & $57 \quad(14.5)$ & $11 \quad(6.2)$ & $0.0043^{a}$ & $(7.6)$ & $0.0498^{a}$ & N/A & N/A \\
\hline ANK & 0 & 0 & N/A & $(1.7)$ & $0.0098^{a}$ & $(0.5)$ & 0.1516 \\
\hline csDMARD use, $\mathrm{n}(\%)^{\mathrm{b}}$ & $305 \quad(77.8)$ & $127 \quad(71.4)$ & 0.0953 & $93 \quad(78.8)$ & 0.8167 & 152 (79.6) & 0.6250 \\
\hline MTX & $209 \quad(53.3)$ & $93 \quad(52.3)$ & 0.8127 & $71 \quad(60.2)$ & 0.1896 & $108 \quad(56.5)$ & 0.4627 \\
\hline Sulfasalazine & $22 \quad(5.6)$ & $18 \quad(10.1)$ & 0.0513 & $(9.3)$ & 0.1510 & $20 \quad(10.5)$ & 0.0332 \\
\hline Hydroxychloroquine & $72 \quad(18.4)$ & $39 \quad(21.9)$ & 0.3223 & $26 \quad(22.0)$ & 0.3755 & $38 \quad(19.9)$ & 0.6581 \\
\hline Leflunomide & $74 \quad(18.9)$ & $24 \quad(13.5)$ & 0.1137 & $20 \quad(17.0)$ & 0.6358 & $34 \quad(17.8)$ & 0.7535 \\
\hline Other csDMARD & $(7.4)$ & $11 \quad(6.2)$ & 0.5978 & $(9.3)$ & 0.4955 & $12 \quad(6.3)$ & 0.6211 \\
\hline \multicolumn{8}{|l|}{ Pain medication use, $\mathrm{n}(\%)$} \\
\hline Opioids & $131 \quad(33.4)$ & $61 \quad(34.3)$ & 0.8420 & $41 \quad(34.8)$ & 0.7892 & $76 \quad(39.8)$ & 0.1313 \\
\hline NSAIDs & $214 \quad(54.6)$ & $103 \quad(57.9)$ & 0.4660 & $58 \quad(49.2)$ & 0.2991 & $85 \quad(44.5)$ & 0.0222 \\
\hline Corticosteroids & $275 \quad(70.2)$ & $117 \quad(65.7)$ & 0.2910 & $87 \quad(73.7)$ & 0.4531 & $136 \quad(71.2)$ & 0.7939 \\
\hline \multicolumn{8}{|l|}{ Top 5 medications, $\mathrm{n}(\%)$} \\
\hline Prednisone & $237 \quad(60.5)$ & $103 \quad(57.9)$ & 0.5585 & $79 \quad(67.0)$ & 0.2030 & $124 \quad(64.9)$ & 0.2977 \\
\hline MTX sodium & $206 \quad(52.6)$ & $93 \quad(52.3)$ & 0.9463 & $71 \quad(60.2)$ & 0.1452 & $108 \quad(56.5)$ & 0.3640 \\
\hline Acetaminophen/hydrocodone bitartrate & $157 \quad(40.1)$ & $74 \quad(41.6)$ & 0.7316 & $54 \quad(45.8)$ & 0.2694 & $90 \quad(47.1)$ & 0.1050 \\
\hline Folic acid & $144 \quad(36.7)$ & $70 \quad(39.3)$ & 0.5538 & $52(44.1)$ & 0.1511 & $72 \quad(37.7)$ & 0.8215 \\
\hline Azithromycin & $87 \quad(22.2)$ & $50 \quad(28.1)$ & 0.1268 & $26 \quad(22.0)$ & 0.9707 & $45 \quad(23.6)$ & 0.7114 \\
\hline
\end{tabular}

asignificant at 0.05 after applying the Hochberg procedure.

bPatients could receive $>1$ csDMARD.

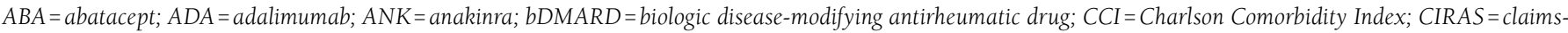
based index of RA severity; $c S D M A R D=$ conventional synthetic disease-modifying antirheumatic drug; $C Z P=$ certolizumab pegol; ETN=etanercept; $G O L=$ golimumab; IFX=infliximab; $M T X=$ methotrexate; $N / A=$ not applicable; NSAID = nonsteroidal anti-inflammatory drug; $R T X=$ rituximab; $S D=$ standard deviation; TCZ = tocilizumab; TNFi $=$ tumor necrosis factor inhibitor. 
Evaluation of Real-World Experience with Tofacitinib Compared with Adalimumab, Etanercept, and Abatacept in RA Patients with 1 Previous Biologic DMARD: Data from a U.S. Administrative Claims Database

FIGURE 2 All-Cause Total, Pharmacy, and Medical Costs in the 12-Month Pre-index and Post-index Periods for Patients Receiving Tofacitinib, ADA, ETN, and ABA

\section{A. Twelve-Month Pre-index Period}

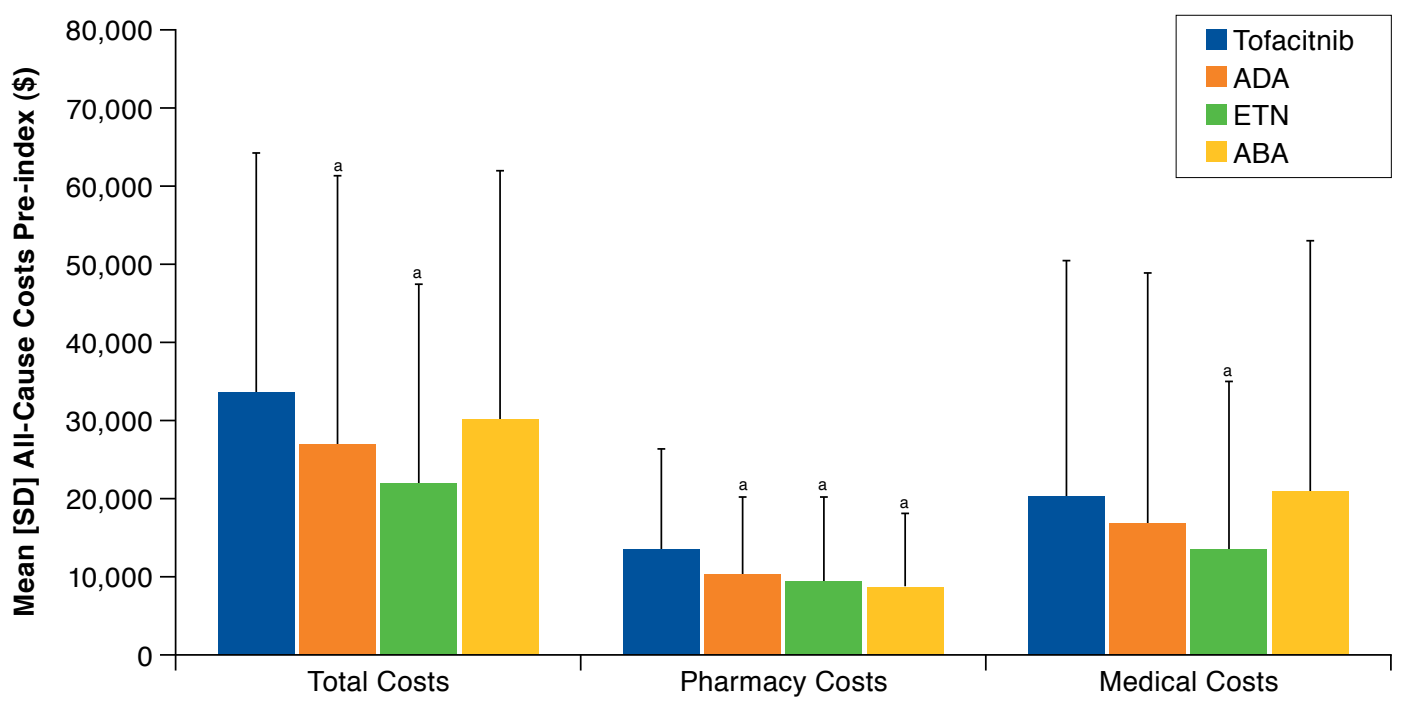

\section{B. Twelve-Month Post-index Period}

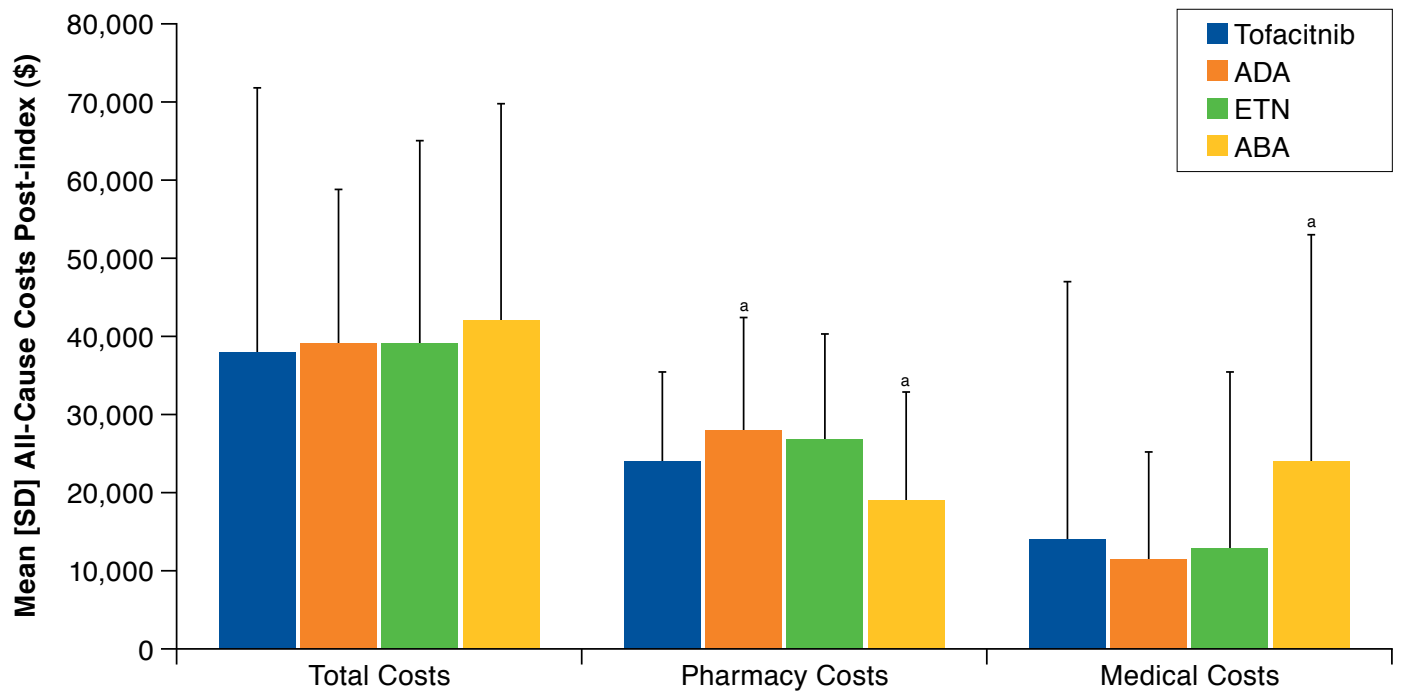

Note: Claims at index were considered as post-index costs. All-cause costs included ambulatory and ER visits, inpatient admissions, and prescriptions for any reason. a Significant at 0.05 after applying the Hochberg procedure.

$A B A=$ abatacept $; A D A=$ adalimumab; ER=emergency room; ETN=etanercept; SD=standard deviation.

ABA (43.5\%; 83/191) were persistent during the 12-month post-index period. Mean persistence (days) was not significantly different for tofacitinib (219.9) versus ADA (221.3), ETN (232.9), and ABA (232.6). Differences remained nonsignificant in adjusted analyses. A greater proportion of patients receiving $\mathrm{ADA}(10.1 \% ; 18 / 178 ; P=0.0263)$ and $\operatorname{ETN}(10.2 \%$; $12 / 118 ; P=0.0466)$ switched early to tofacitinib or a nonindex bDMARD versus those receiving tofacitinib (5.1\%; 20/392); neither were significant when adjusting for multiple comparisons. A similar proportion of patients (range: 13.6\%-18.9\%) had 
Evaluation of Real-World Experience with Tofacitinib Compared with Adalimumab, Etanercept, and Abatacept in RA Patients with 1 Previous Biologic DMARD: Data from a U.S. Administrative Claims Database

TABLE 2 RA-Related Total, Pharmacy, and Medical Costs in 12-Month Pre-index and Post-index Periods

\begin{tabular}{|c|c|c|c|c|c|c|c|}
\hline & $\begin{array}{c}\text { Tofacitinib } \\
(\mathrm{n}=392)\end{array}$ & $\begin{array}{c}\text { ADA } \\
(n=178)\end{array}$ & $\begin{array}{c}P \text { Value } \\
\text { ADA vs. } \\
\text { Tofacitinib }\end{array}$ & $\begin{array}{c}\text { ETN } \\
(n=118)\end{array}$ & $\begin{array}{c}P \text { Value } \\
\text { ETN vs. } \\
\text { Tofacitinib }\end{array}$ & $\begin{array}{c}\text { ABA } \\
(n=191)\end{array}$ & $\begin{array}{c}P \text { Value } \\
\text { ABA vs. } \\
\text { Tofacitinib }\end{array}$ \\
\hline \multicolumn{8}{|c|}{ A. Twelve-Month Pre-index Period } \\
\hline $\begin{array}{l}\text { Total costs, } \\
\text { mean }[S D] / \text { median, \$ }\end{array}$ & $\begin{array}{c}20,784 \\
{[20,727] / 16,623}\end{array}$ & $\begin{array}{c}12,581 \\
{[14,115] / 8,211}\end{array}$ & $<0.0001^{\mathrm{a}}$ & $\begin{array}{c}9,750 \\
{[11,455] / 4,925}\end{array}$ & $<0.0001^{\mathrm{a}}$ & $\begin{array}{c}13,490 \\
{[15,392] / 7,471}\end{array}$ & $<0.0001^{\mathrm{a}}$ \\
\hline $\begin{array}{l}\text { Pharmacy costs, } \\
\text { mean [SD]/median, \$ }\end{array}$ & $\begin{array}{c}10,014 \\
{[11,100] / 6,634}\end{array}$ & $\begin{array}{c}7,299 \\
{[8,875] / 2,298} \\
\end{array}$ & $0.0019^{a}$ & $\begin{array}{c}5,460 \\
{[9,105] / 380}\end{array}$ & $<0.0001^{\mathrm{a}}$ & $\begin{array}{c}5,076 \\
{[7,780] / 349}\end{array}$ & $<0.0001^{a}$ \\
\hline bDMARDs & $\begin{array}{c}9,706 \\
{[11,088] / 6,401} \\
\end{array}$ & $\begin{array}{c}7,088 \\
{[8,858] / 2,200}\end{array}$ & $0.0027^{\mathrm{a}}$ & $\begin{array}{c}5,202 \\
{[9,133] / 0}\end{array}$ & $<0.0001^{\mathrm{a}}$ & $\begin{array}{c}4,872 \\
{[7,759] / 0}\end{array}$ & $<0.0001^{\mathrm{a}}$ \\
\hline Select csDMARDs ${ }^{\mathrm{b}}$ & $272[373] / 144$ & $173[234] / 101$ & $0.0001^{\mathrm{a}}$ & $153[178] / 100$ & $<0.0001^{\mathrm{a}}$ & $193[263] / 96$ & $0.0033^{\mathrm{a}}$ \\
\hline $\begin{array}{l}\text { Other csDMARD } \\
\text { medications }\end{array}$ & $\begin{array}{c}37 \\
{[208] / 0}\end{array}$ & $\begin{array}{c}38 \\
{[224] / 0}\end{array}$ & 0.9464 & $\begin{array}{c}104 \\
{[485] / 0}\end{array}$ & 0.1438 & $\begin{array}{c}11 \\
{[70] / 0}\end{array}$ & 0.0278 \\
\hline $\begin{array}{l}\text { bDMARD administration } \\
\text { costs, mean [SD]/median, } \$\end{array}$ & $\begin{array}{c}4,496 \\
{[13,331] / 0} \\
\end{array}$ & $\begin{array}{c}608 \\
{[3,074] / 0}\end{array}$ & $<0.0001^{\mathrm{a}}$ & $\begin{array}{c}803 \\
{[2,709] / 0} \\
\end{array}$ & $<0.0001^{\mathrm{a}}$ & $\begin{array}{c}1,655 \\
{[6,240] / 0}\end{array}$ & $0.0005^{\mathrm{a}}$ \\
\hline $\begin{array}{l}\text { Medical costs, } \\
\text { mean }[S D] / \text { median, \$ }\end{array}$ & $\begin{array}{c}10,770 \\
{[20,552] / 1,589}\end{array}$ & $\begin{array}{c}5,282 \\
{[11,634] / 1,087}\end{array}$ & $<0.0001^{a}$ & $\begin{array}{c}4,290 \\
{[7,448] / 1,110}\end{array}$ & $<0.0001^{a}$ & $\begin{array}{c}8,414 \\
{[14,880] / 1,388}\end{array}$ & 0.1158 \\
\hline Inpatient admissions & $2,693[12,012] / 0$ & $1,702[7,735] / 0$ & 0.2382 & $1,466[5,505] / 0$ & 0.1214 & $2,188[8,655] / 0$ & 0.5628 \\
\hline Ambulatory visits & $\begin{array}{c}8,077 \\
{[16,495] / 1,499} \\
\end{array}$ & $\begin{array}{c}3,580 \\
{[7,437] / 1,015} \\
\end{array}$ & $<0.0001^{\mathrm{a}}$ & $\begin{array}{c}2,824 \\
{[5,191] / 1,006} \\
\end{array}$ & $<0.0001^{\mathrm{a}}$ & $\begin{array}{c}6,226 \\
{[12,651] / 1,262} \\
\end{array}$ & 0.1355 \\
\hline Office visits & $601[728] / 494$ & 518 [399]/432 & 0.0793 & $536[395] / 468$ & 0.2106 & $545[381] / 458$ & 0.2273 \\
\hline Outpatient visits & $7,476[16,418] / 810$ & $3,063[7,325] / 437$ & $<0.0001^{\mathrm{a}}$ & $2,288[5,084] / 465$ & $<0.0001^{\mathrm{a}}$ & $5,680[12,582] / 686$ & 0.1455 \\
\hline ER visits & $123[927] / 0$ & $194[1,340] / 0$ & 0.5256 & $198[853] / 0$ & 0.4313 & $99[792] / 0$ & 0.7435 \\
\hline Urgent health care visits & 0 & 0 & N/A & $4[34] / 0$ & 0.2621 & $16[208] / 0$ & 0.2901 \\
\hline Home health care visits & $14[136] / 0$ & 0 & 0.0465 & $11[74] / 0$ & 0.7568 & $50[405] / 0$ & 0.2238 \\
\hline $\begin{array}{l}\text { Other unclassified } \\
\text { services }\end{array}$ & $\begin{array}{c}7,339 \\
{[16,393] / 755} \\
\end{array}$ & $\begin{array}{c}2,869 \\
{[7,258] / 421}\end{array}$ & $<0.0001^{\mathrm{a}}$ & $\begin{array}{c}2,079 \\
{[5,043] / 399}\end{array}$ & $<0.0001^{\mathrm{a}}$ & $\begin{array}{c}5,531 \\
{[12,540] / 596} \\
\end{array}$ & 0.1417 \\
\hline \multicolumn{8}{|c|}{ B. Twelve-Month Post-index Period } \\
\hline $\begin{array}{l}\text { Total costs, } \\
\text { mean }[S D] / \text { median, \$ }\end{array}$ & $\begin{array}{c}24,793 \\
{[13,320] / 24,072} \\
\end{array}$ & $\begin{array}{c}28,046 \\
{[13,708] / 27,538}\end{array}$ & $0.0076^{\mathrm{a}}$ & $\begin{array}{c}25,376 \\
{[11,906] / 28,213} \\
\end{array}$ & 0.6696 & $\begin{array}{c}29,800 \\
{[17,357] / 28,791} \\
\end{array}$ & $0.0005^{\mathrm{a}}$ \\
\hline $\begin{array}{l}\text { Pharmacy costs, } \\
\text { mean }[S D] / \text { median, } \$\end{array}$ & $\begin{array}{c}19,804 \\
{[9,467] / 21,186}\end{array}$ & $\begin{array}{c}24,237 \\
{[12,713] / 24,272}\end{array}$ & $<0.0001^{\mathrm{a}}$ & $\begin{array}{c}22,140 \\
{[10,651] / 25,251} \\
\end{array}$ & $0.0229^{a}$ & $\begin{array}{c}15,282 \\
{[13,657] / 13,692} \\
\end{array}$ & $<0.0001^{\mathrm{a}}$ \\
\hline Tofacitinib & $\begin{array}{c}16,318 \\
{[9,372] / 16,602}\end{array}$ & 0 & $<0.0001^{\mathrm{a}}$ & 0 & $<0.0001^{\mathrm{a}}$ & 0 & $<0.0001^{\mathrm{a}}$ \\
\hline bDMARDs & $\begin{array}{c}3,178 \\
{[7,782] / 0} \\
\end{array}$ & $\begin{array}{c}23,915 \\
{[12,653] / 24,041} \\
\end{array}$ & $<0.0001^{\mathrm{a}}$ & $\begin{array}{c}21,789 \\
{[10,543] / 24,778}\end{array}$ & $<0.0001^{\mathrm{a}}$ & $\begin{array}{c}14,959 \\
{[13,646] / 13,604} \\
\end{array}$ & $<0.0001^{\mathrm{a}}$ \\
\hline Select csDMARDs ${ }^{\mathrm{b}}$ & $247[383] / 84$ & $274[529] / 113$ & 0.5432 & $220[290] / 127$ & 0.4100 & $266[332] / 128$ & 0.5365 \\
\hline $\begin{array}{l}\text { Other csDMARD } \\
\text { medications }\end{array}$ & $\begin{array}{c}60 \\
{[293] / 0} \\
\end{array}$ & $\begin{array}{c}47 \\
{[231] / 0}\end{array}$ & 0.5611 & $\begin{array}{c}131 \\
{[596] / 0}\end{array}$ & 0.2146 & $\begin{array}{c}56 \\
{[391] / 0} \\
\end{array}$ & 0.8961 \\
\hline $\begin{array}{l}\text { bDMARD administration } \\
\text { costs, mean }[\mathrm{SD}] / \text { median, } \$\end{array}$ & $\begin{array}{c}1,184 \\
{[5,811] / 0}\end{array}$ & $\begin{array}{c}1,050 \\
{[5,510] / 0}\end{array}$ & 0.7954 & $\begin{array}{c}1,144 \\
{[4,645] / 0}\end{array}$ & 0.9382 & $\begin{array}{c}9,888 \\
{[16,011] / 0} \\
\end{array}$ & $<0.0001^{\mathrm{a}}$ \\
\hline $\begin{array}{l}\text { Medical costs, } \\
\text { mean }[S D] / \text { median, } \$\end{array}$ & $4,989[11,366] / 965$ & $3,809[7,997] / 908$ & 0.1558 & $3,236[7,350] / 730$ & 0.0490 & $14,518[20,189] / 4,021$ & $<0.0001^{\mathrm{a}}$ \\
\hline Inpatient admissions & $1,481[7,171] / 0$ & $833[4,190] / 0$ & 0.1770 & $1,305[5,872] / 0$ & 0.7865 & $2,103[11,693] / 0$ & 0.4998 \\
\hline Ambulatory visits & $3,508[8,951] / 878$ & $2,976[6,955] / 882$ & 0.4413 & $1,931[4,345] / 703$ & $0.0093^{\mathrm{a}}$ & $12,415[17,453] / 2,604$ & $<0.0001^{\mathrm{a}}$ \\
\hline Office visits & $565[601] / 466$ & $484[355] / 417$ & 0.0452 & $464[367] / 410$ & 0.0261 & $529[353] / 471$ & 0.3642 \\
\hline Outpatient visits & $\begin{array}{c}2,942 \\
{[8,818] / 324}\end{array}$ & $\begin{array}{c}2,492 \\
{[6,810] / 331}\end{array}$ & 0.5061 & $\begin{array}{c}1,467 \\
{[4,186] / 213}\end{array}$ & $0.0126^{\mathrm{a}}$ & $\begin{array}{c}11,886 \\
{[17,394] / 2,103}\end{array}$ & $<0.0001^{\mathrm{a}}$ \\
\hline ER visits & $181[1,355] / 0$ & $96[603] / 0$ & 0.2995 & $68[382] / 0$ & 0.1412 & $77[440] / 0$ & 0.1672 \\
\hline Urgent health care visits & $0[9] / 0$ & $3[27] / 0$ & 0.1611 & $2[22] / 0$ & 0.3391 & $172[2,374] / 0$ & 0.3199 \\
\hline Home health care visits & $12[101] / 0$ & $1[10] / 0$ & 0.0402 & $9[99] / 0$ & 0.8160 & $14[180] / 0$ & 0.8548 \\
\hline $\begin{array}{l}\text { Other unclassified } \\
\text { services }\end{array}$ & $\begin{array}{c}2,750 \\
{[8,299] / 303}\end{array}$ & $\begin{array}{c}2,395 \\
{[6,803] / 304}\end{array}$ & 0.5910 & $\begin{array}{c}1,390 \\
{[4,180] / 176}\end{array}$ & $0.0173^{a}$ & $\begin{array}{c}11,795 \\
{[17,405] / 2,025}\end{array}$ & $<0.0001^{\mathrm{a}}$ \\
\hline
\end{tabular}

a Significant at 0.05 after applying the Hochberg procedure.

${ }^{b} \mathrm{MTX}$, sulfasalazine, hydrochloroquine, and leflunomide.

$A B A=$ abatacept; $A D A=$ adalimumab; $b D M A R D=$ biologic disease-modifying antirheumatic drug; csDMARD = conventional synthetic disease-modifying antirheumatic drug; $E R=$ emergency room; $E T N=$ etanercept $; M T X=$ methotrexate; $R A=$ rheumatoid arthritis; $S D=$ standard deviation. 
delayed switch to tofacitinib or a nonindex bDMARD. Overall switch rate was similar for patients receiving tofacitinib (25.0\%) versus ADA (27.5\%), ETN (23.7\%), and ABA (24.6\%); pre-index bDMARD and TNFi use were associated with significantly higher rates of switching in adjusted analyses.

Mean (standard deviation) PDC was similar for patients receiving tofacitinib $(0.55[0.30])$, ADA (0.57 [0.30]), and ETN (0.59 [0.31]) but was significantly lower for ABA patients (0.44 [0.39]; $P=0.0003$ ) versus tofacitinib patients. The proportion of patients with PDC $\geq 0.8$ ranged from $29.1 \%$ to $36.4 \%$. In adjusted analyses, ABA use was associated with a significantly lower mean PDC versus tofacitinib; however, there was no significant difference versus tofacitinib in the proportion of patients with $\mathrm{PDC} \geq 0.8$ in unadjusted and adjusted analyses. Increasing age was associated with significantly higher PDC (as was commercial insurance coverage) and proportion of patients with $\mathrm{PDC} \geq 0.8$.

Dose escalation was more common among ADA patients (15.3\%; 26/170) versus tofacitinib patients (7.4\%; 27/366; $P=0.0043)$. There were no significant differences in dose escalation between tofacitinib patients and ETN $(2.7 \% ; 3 / 110)$ and $\operatorname{ABA}(7.0 \%$; 13/186) patients.

\section{Costs}

Twelve-Month Pre-index Costs. Tofacitinib patients had greater pre-index all-cause total costs versus ADA and ETN patients $(P<0.05)$, medical costs versus ETN patients $(P=0.0064)$, and pharmacy costs versus patients receiving bDMARDs (all $P<0.05$; Figure $2 \mathrm{~A}$ ).

Tofacitinib patients had greater pre-index RA-related total and pharmacy costs versus patients receiving bDMARDs (all $P<0.05$ ) and greater pre-index RA-related medical costs versus ADA and ETN patients (both $P<0.0001$; Table $2 \mathrm{~A}$ ). Pharmacy costs comprised $48.2 \%$ of the total RA-related costs for tofacitinib patients versus $58.0 \%, 56.0 \%$, and $37.6 \%$ of the RA-related total costs for ADA, ETN, and ABA patients, respectively. Most RA-related pharmacy costs were attributed to the cost of bDMARDs and their administration (Table 2A). RA-related medical costs comprised $51.8 \%$ of the total costs for tofacitinib patients versus $42.0 \%, 44.0 \%$, and $62.4 \%$ of the total costs for ADA, ETN, and ABA patients, respectively. Most RA-related medical costs were attributed to ambulatory visits (Table 2A).

Twelve-Month Post-index Costs. Total unadjusted post-index mean all-cause costs were similar for patients receiving tofacitinib and bDMARDs. Post-index all-cause pharmacy costs for tofacitinib patients were lower versus ADA patients but higher versus ABA patients (both $P<0.001$ ), while post-index allcause medical costs were lower for tofacitinib patients versus ABA patients $(P=0.0009$; Figure 2B). In adjusted analyses, the total post-index mean (95\% confidence interval $[\mathrm{CI}]$ ) all-cause cost was significantly lower for tofacitinib versus ADA, ETN, and ABA (Table 3A). While CCI score, $\geq 3$ csDMARDs pre-index, rheumatologist visits pre-index, and total costs pre-index were associated with significantly higher allcause total costs, the South and West regions were associated with lower costs (vs. Northeast region; Table 3B).

Tofacitinib patients had significantly lower unadjusted postindex RA-related total costs versus ADA and ABA patients, significantly lower pharmacy costs versus ADA and ETN patients, and significantly lower medical costs versus ABA patients (all $\mathrm{P}<0.05$; Table 2B). Post-index RA-related pharmacy costs accounted for most of the total costs for patients receiving tofacitinib (79.9\%), ADA (86.4\%), and ETN (87.2\%) and $>50 \%$ of the total cost for ABA patients. Most RA-related pharmacy costs for tofacitinib patients were attributed to the cost of tofacitinib followed by the cost of bDMARDs and their administration (Table 2B). For patients receiving bDMARDs, RA-related costs associated with bDMARDs and their administration comprised most of the pharmacy costs (Table 2B). Most post-index RA-related medical costs for patients receiving tofacitinib and bDMARDs were attributed to ambulatory visits (Table 2B).

In adjusted analyses, total post-index mean (95\% CI) RA-related costs were significantly higher for ADA, ETN, and $A B A$ versus tofacitinib (Table 3A). Furthermore, commercial coverage, rheumatologist visits pre-index, and RA-related costs preindex were associated with significantly higher RA-related total costs, while the North Central region and the South (Northeast as reference) were associated with lower costs (Table 3B).

\section{Discussion}

Tofacitinib is the first oral JAK inhibitor approved for RA. Although a network meta-analysis of randomized controlled trial data demonstrated similar efficacy for tofacitinib versus bDMARDs in improving RA signs and symptoms in patients with inadequate response to TNF inhibitors, ${ }^{34} \mathrm{lim}$ ited real-world data are available. In this study, we compared patient characteristics, treatment patterns, and costs in RA patients receiving tofacitinib versus ADA, ETN, and ABA, after 1 bDMARD in a U.S. claims database. Tofacitinib persistence and adherence were at least comparable versus bDMARDs, with lower adjusted total RA-related costs. However, significant differences between treatment cohorts, particularly with regard to 12-month pre-index bDMARD use and RA-related costs in favor of bDMARDs, were observed, which may suggest channeling bias for tofacitinib. Future analyses will be required, ideally under similar access and prescribing conditions and incorporating data with clinical information, to assess severity and outcomes.

Although this analysis focused on patients who received 1 previous bDMARD, tofacitinib patients were more likely to receive a bDMARD in the 12 -month pre-index period versus 
Evaluation of Real-World Experience with Tofacitinib Compared with Adalimumab, Etanercept, and Abatacept in RA Patients with 1 Previous Biologic DMARD: Data from a U.S. Administrative Claims Database

TABLE 3 Adjusted Analyses for All-Cause and RA-Related Total Costs in the 12-Month Post-index Period Tofacitinib

ADA

ETN

ABA

A. Adjusted 12-Month Post-index All-Cause and RA-Related Total Costs

All-cause total costs, \$

Mean

95\% CI

36,945

$33,462-40,790$

42,221

42,219

43,042

RA-related total costs, \$

Mean

$95 \% \mathrm{CI}$

\begin{tabular}{c|c}
23,568 & 29,278 \\
\hline $21,258-26,130$ & $26,058-32,896$ \\
\hline
\end{tabular}

All Cause

Coefficient $\quad$ P Value

\begin{tabular}{l|l}
$37,156-47,970$ & $38,483-48,141$ \\
\hline
\end{tabular}

\section{Coefficient}

\begin{tabular}{l|r}
\hline B. Coefficients from Generalized Linear Models for Analysis of 12-Month Post-Index \\
\hline Intercept & 9.8802
\end{tabular}

Age

0.0042

\begin{tabular}{|r} 
All-Cause and RA \\
\hline 0.0001 \\
0.0515
\end{tabular}

26,885

23,594-30,634

30,477

Region

Northeast (reference)

North Central

South

West

Unknown

-0.1134
-0.2145
-0.1844
0.0595

$\mid$

\begin{tabular}{|r|r|} 
& \\
\hline & 0.0515 \\
\hline 0.0001 \\
0.0032 \\
\hline 0.6731
\end{tabular}

$\mid$

Coefficient

27,154-34,206

Sex

1 (reference)

2

Insurance type of index claim

0.0183

$\mid$

0.7091

9

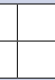

Total Costs

9.6764

0.0019

$P$ Value

Medicare (reference)

Commercial

Length of pre-index period

Quan-CCI score during 12 months pre-index

CIRAS score during 12 months pre-index

Number of days between first RA diagnosis and index

\begin{tabular}{c|c} 
& \\
\hline-0.0162 & 0.7860 \\
\hline 0.0000 & 0.9555 \\
\hline 0.0473 & 0.0086 \\
\hline 0.0114 & 0.5176 \\
\hline $1.95 \times 10^{-5}$ & 0.7681
\end{tabular}

\begin{tabular}{|r|r|}
\hline & -0.1275 \\
\hline & -0.2191 \\
\hline & -0.1197 \\
\hline & 0.0167 \\
\hline
\end{tabular}

\begin{tabular}{r|r}
\hline$<0.0001$ \\
\hline
\end{tabular}

csDMARD count during 12 months pre-index

\begin{tabular}{l}
0 (re \\
\hline 1 \\
\hline 23
\end{tabular}

(reference)

$\geq 3$

Index medication

Tofacitinib (reference)

ADA

ETN

ABA

(reference)

\begin{tabular}{|l|l|}
\hline & \\
\hline
\end{tabular}

$1.95 \times 10^{-5}$

0.7681

\begin{tabular}{l|l} 
& \\
0.0095 &
\end{tabular}

0.8515

Use of $\geq 1$ select csDMARD during

90 days post-index

No (reference)

Yes

Number of rheumatologist visits during 12 months pre-index

Unique number of opioid prescriptions during 12 months pre-index

\begin{tabular}{l}
0.0290 \\
\hline 0.0523 \\
\hline 0.1834
\end{tabular}

$\mid$

\begin{tabular}{|l|l|} 
& \\
0.5586 & \\
0.3797 & \\
\hline 0.0404 & \\
\hline
\end{tabular}

\begin{tabular}{|c|c|}
\hline & 0.1306 \\
\hline 0.0000 \\
0.0344 \\
\hline-0.0037 \\
\hline $8.16 \times 10^{-5}$ \\
\hline
\end{tabular}

\begin{tabular}{|l|l}
\hline & \\
\hline & 0.0351 \\
0.0001 \\
0.0656 \\
0.9088
\end{tabular}

TNFi use during whole database pre-index

No (reference)

Yes

bDMARD use before 12 months pre-index

No (reference)

Yes

Total costs during 12 months pre-index

\begin{tabular}{|l|l|l} 
& & \\
\hline 0.1335 & $0.0065^{\mathrm{a}}$ & \\
\hline 0.1334 & $0.0174^{\mathrm{a}}$ & \\
\hline 0.1527 & $0.0017^{\mathrm{a}}$ & \\
\hline
\end{tabular}

2

\begin{tabular}{l|l} 
& \\
0.0599 & \\
0.0321 & \\
\hline 0.1065 &
\end{tabular}

\begin{tabular}{|l|l}
\hline & \\
\hline & 0.0368 \\
\hline & 0.6813 \\
\hline & 0.0549 \\
\hline
\end{tabular}

0.2308

a Significant at 0.05 after applying the Hochberg procedure.

$A B A=$ abatacept $A D A=$ adalimumab; $b D M A R D=$ biologic disease-modifying antirheumatic drug; $C C I=$ Charlson Comorbidity Index; $C I=$ confidence interval;

CIRAS = claims-based index of RA severity; csDMARD = conventional synthetic disease-modifying antirheumatic drug; ETN=etanercept; RA=rheumatoid arthritis;

$\mathrm{TNFi}=$ tumor necrosis factor inhibitor . 
bDMARD patients, potentially indicating differences in disease severity between groups; bDMARD patients were possibly more likely to have previous treatment response or remission. Disease severity was assessed by CIRAS score, in the absence of outcome measures; however, the validity of CIRAS as a robust proxy has been questioned. ${ }^{35}$ Pre-index RA-related costs may be a better indicator of severity and were significantly higher for tofacitinib. This finding is interesting given the significantly higher unadjusted medical costs versus ADA and ETN. In addition, tofacitinib patients had higher previous prescription and administration costs and an increased likelihood of receiving a non-TNFi bDMARD, further suggesting more complicated cases compared with the bDMARD groups. Thus, there is likely significant channeling bias with tofacitinib, but this would have biased against tofacitinib.

A substantial proportion of patients started monotherapy with tofacitinib or a bDMARD, despite most receiving a csDMARD in the 12-month pre-index period. A greater proportion of patients received tofacitinib monotherapy versus bDMARD monotherapy, and among these monotherapy patients, tofacitinib was associated with the lowest proportion of patients who initiated csDMARDs in the 12-month follow-up period. This is supported by data from the Corrona registry, where $43.2 \%$ of tofacitinib patientsreceived monotherapyversus $27.3 \%$ of patients receiving bDMARDs. ${ }^{36}$ Use of bDMARDs with csDMARDs may be more likely following reports of enhanced efficacy with some TNFi when combined with MTX. ${ }^{9-13}$ TCZ has demonstrated comparable safety and efficacy as monotherapy or combination therapy, ${ }^{37-39}$ with a trend towards better outcomes with combination therapy in some cases. ${ }^{37,40,41} \mathrm{TCZ}$ monotherapy has also been linked with lower treatment retention versus combination therapy. ${ }^{41}$

Persistence was similar for patients receiving tofacitinib and bDMARDs and comparable with previous data in patients receiving bDMARDs and non-RA therapies., ${ }^{42,43}$ Adherence, evaluated using PDC, was similar to previous reports for TNFi in RA. Mean PDC values of 0.57 for ETN and 0.64 for IFX have been reported in Medicaid patients, ${ }^{44}$ while adherence of 0.52 has been reported in an inception cohort of patients with pharmacy claims for ETN and ADA in an insurance claims database of self-insured employer health plans. ${ }^{45}$ Adherence was consistent with the range (35\%-72\%) reported by Yeaw et al. (2009) in their analysis of pharmacy claims across 6 chronic medication classes. ${ }^{43}$

Furthermore, mean PDC values were similar for patients receiving tofacitinib, ADA, and ETN but significantly lower with ABA versus tofacitinib. Although not possible to discern here, differences in adherence may be attributed to several factors, including safety and efficacy profiles, frequency and route of administration, and cost of treatment. "Inadequate therapy behaviour," associated with increased risk of RA flare, was observed in patients with RA who had achieved remission with DMARDs and had mild or no disease activity/disability. ${ }^{46}$ Differences in route of administration exist between ADA, ETN, and ABA. ${ }^{16-18}$ Whereas ADA and ETN are administered subcutaneously, ABA is often administered by intravenous infusion and was approved for subcutaneous administration in 2011. ${ }^{17}$ Mean RA-related costs for bDMARD administrations over the 12-month post-index follow-up for the ABA cohort was $\$ 10,000$ versus approximately $\$ 1,000$ for comparators. Oral and subcutaneous routes of administration offer patients more flexibility and convenience than intravenous infusion, since medications can be administered at home at a time selected by the patient and could lead to improved adherence. In a choice-based conjoint survey mailed to 1,400 Humana members with RA, the most frequently selected route of administration was oral (0.754), followed by self-injection (0.492) and intravenous infusion (0.263). ${ }^{47}$ In addition, a single center British study of $100 \mathrm{RA}$ patients reported that those receiving TNFi preferred subcutaneous administration over intramuscular or intravenous administration and preferred home administration versus an outpatient/inpatient setting. ${ }^{48}$

Literature comparing adherence and persistence for oral versus injectable and infusible products is challenging and limited. Some evidence exists that discontinuation or nonadherence rates are higher for the most common infusible bDMARD, IFX, versus self-injectable bDMARDs (e.g., ETN and $\mathrm{ADA}^{49-52}$ ). Other literature report similar or better treatment persistence/adherence with IFX. ${ }^{53-55}$ The evaluation of subcutaneous versus oral versions of MTX found no difference in the proportion of patients discontinuing treatment at 1 year. ${ }^{56}$ In other therapeutic areas previously dominated by injectable therapies where oral options are being introduced, there is emerging evidence, sometimes conflicting, regarding the benefits of oral therapy on treatment persistence/ adherence. ${ }^{57-59}$ Route of administration may play a role in RA treatment initiation. Primary nonadherence to bDMARDs has been reported in nearly $40 \%$ of RA patients within 6 months after being prescribed a bDMARD ${ }^{60}$ Thus, understanding the impact of route of administration on DMARD initiation and adherence is critical.

Dose escalation was relatively uncommon among tofacitinib-treated patients, which is not surprising given that $5 \mathrm{mg}$ BID is the approved dose in the United States, and dose escalation is not permitted..$^{24}$ Dose escalation was significantly more common for patients receiving ADA versus tofacitinib, which is consistent with the fact that it is label permitted. ${ }^{18}$

Total adjusted RA-related costs in the 12-month post-index period were significantly lower for patients receiving tofacitinib versus bDMARDs, despite significantly higher pre-index costs. Total RA-related costs post-index were highest for patients receiving ABA. These results dovetail with data on TNFi cycling. ${ }^{61}$ 


\section{Limitations}

This study has some limitations to consider. Claims data are collected for payment processing only; therefore, a claim does not indicate that the patient took the medication. Similarly, a "no claim" period may not indicate nonadherence but temporary treatment discontinuation. Coding errors could also affect the ability to detect all relevant health care resource use. Furthermore, there were no medical history, clinical severity, outcome indicators, or reasons for discontinuation in these claims data. Claims analyses of bDMARDs in RA typically require a 6- to 12-month pre-index enrollment period. 31,32,44,62-64 While we required $\geq 12$ months pre-index enrollment, we found that a significant proportion of RA patients had bDMARDs before the 1-year pre-index period, thus, we used all available history. Because we did not require enrollment beyond 1 year, and the data were available on January 1, 2009, it is possible that we have underestimated previous bDMARD use across treatment groups. However, it should be noted that mean (SD) enrollment was 1,116 (353) days, and there were no significant differences across treatment groups.

In assessing treatment patterns, an adjustment for days supply was implemented to address the inaccuracy of the days supply field and varying interpretation of the days supply for monthly prescriptions for injectable versus oral therapies. Similar issues have been described in other therapeutic areas. The most frequent days supply value for an oral second-generation antipsychotic (SGA) is 30 days versus 28 days for a longacting injectable SGA. In this example, a 2-day discrepancy in days supply for the long-acting SGA resulted in significantly fewer patients with a treatment gap. ${ }^{65}$ In our study, this issue was addressed by adjusting the days supply to the nearest 28-day supply. This had little impact on the bDMARDs, since $94 \%$ of those prescriptions were 28-day or 84-day supplies and not requiring adjustment. In contrast, for tofacitinib, the adjusted days supply would be less than using actual days supply for which $98 \%$ of tofacitinib prescriptions were 30-day or 90-day supplies, which likely lowered the persistence and adherence values to make a more robust comparison. As a newly available treatment option, tofacitinib prescriptions could be influenced by channeling bias, whereby patients are more likely to receive tofacitinib if they have previously had an inadequate response/ intolerance to csDMARDs and bDMARDs. Furthermore, as we selected tofacitinib patients first, we may have introduced selection bias with regard to bDMARD experience.

\section{Conclusions}

In this cohort of RA patients with 1 previous bDMARD, more patients initiating treatment with tofacitinib versus the bDMARDs received monotherapy and stayed on monotherapy $(P<0.05$ for ABA). Persistence and adherence were generally similar for tofacitinib versus bDMARDs. Total adjusted RA-related costs post-index were lower for tofacitinib versus
bDMARDs, despite significantly higher pre-index costs. While these data support tofacitinib, further analysis is warranted given the significant differences observed between treatment groups, which suggests channeling bias.

\section{Authors}

JAMES HARNETT, PharmD, MS, and CONNIE CHEN, PharmD, Pfizer, New York, New York; ROBERT GERBER, PharmD, and DAVID GRUBEN, PhD, Pfizer, Groton, Connecticut; and ANDREW S. KOENIG, DO, Pfizer, Collegeville, Pennsylvania.

AUTHOR CORRESPONDENCE: James Harnett, PharmD, MS, Pfizer, 235 E. 42nd St., New York, NY 10017. Tel.: 212.733.3753; Fax: 646.563.1568; E-mail: james.harnett@pfizer.com.

\section{DISCLOSURES}

This study was sponsored by Pfizer. Harnett, Gerber, Gruben, Koenig, and Chen are employees and shareholders of Pfizer.

Some data reported in this manuscript have been previously presented at the Academy of Managed Care Nexus 2015; Orlando, Florida; October 26-29, 2015, and was submitted in abstract form to the European League Against Rheumatism Congress; London, United Kingdom; June 8-11, 2016.

All authors were involved in the conception and design of this study. Harnett and Gruben were involved in data collection and analysis. All authors interpreted the data, critically reviewed and revised the manuscript, and read and approved the final manuscript.

\section{ACKNOWLEDGMENTS}

Editorial support, under the direction of the authors, was provided by Karen Irving, Complete Medical Communications, and funded by Pfizer.

\section{REFERENCES}

1. Myasoedova E, Crowson CS, Kremers HM, Therneau TM, Gabriel SE. Is the incidence of rheumatoid arthritis rising? Results from Olmsted County, Minnesota, 1955-2007. Arthritis Rheum. 2010;62(6):1576-82.

2. Birnbaum H, Pike C, Kaufman R, Marynchenko M, Kidolezi Y, Cifaldi M. Societal cost of rheumatoid arthritis patients in the U.S. Curr Med Res Opin. 2010;26(1):77-90

3. Kawatkar AA, Jacobsen SJ, Levy GD, Medhekar SS, Venkatasubramaniam KV, Herrinton LJ. Direct medical expenditure associated with rheumatoid arthritis in a nationally representative sample from the medical expenditure panel survey. Arthritis Care Res (Hoboken). 2012;64(11):1649-56.

4. Smolen JS, Breedveld FC, Burmester GR, et al. Treating rheumatoid arthritis to target: 2014 update of the recommendations of an international task force. Ann Rheum Dis. 2016;75(1):3-15. 
5. Singh JA, Saag KG, Bridges SL Jr, et al. 2015 American College of Rheumatology guideline for the treatment of rheumatoid arthritis. Arthritis Rheumatol. 2016;68(1):1-26.

6. Pinals RS, Masi AT, Larsen RA. Preliminary criteria for clinical remission in rheumatoid arthritis. Arthritis Rheum. 1981;24(10):1308-15.

7. Felson DT, Smolen JS, Wells G, et al. American College of Rheumatology/ European League Against Rheumatism provisional definition of remission in rheumatoid arthritis for clinical trials. Arthritis Rheum. 2011;63(3):573-86.

8. Smolen JS, Landewé R, Breedveld FC, et al. EULAR recommendations for the management of rheumatoid arthritis with synthetic and biological disease-modifying antirheumatic drugs: 2013 update. Ann Rheum Dis. 2014;73(3):492-509.

9. Maini RN, Breedveld FC, Kalden JR, et al. Therapeutic efficacy of multiple intravenous infusions of anti-tumor necrosis factor alpha monoclonal antibody combined with low-dose weekly methotrexate in rheumatoid arthritis. Arthritis Rheum. 1998;41(9):1552-63.

10. Breedveld FC, Weisman MH, Kavanaugh AF, et al. The PREMIER study: a multicenter, randomized, double-blind clinical trial of combination therapy with adalimumab plus methotrexate versus methotrexate alone or adalimumab alone in patients with early, aggressive rheumatoid arthritis who had not had previous methotrexate treatment. Arthritis Rheum. 2006;54(1):26-37.

11. van der Heijde D, Klareskog L, Landewé R, et al. Disease remission and sustained halting of radiographic progression with combination etanercept and methotrexate in patients with rheumatoid arthritis. Arthritis Rheum. 2007;56(12):3928-39.

12. Keystone EC, Genovese MC, Klareskog L, et al. Golimumab, a human antibody to tumour necrosis factor \{alpha\} given by monthly subcutaneous injections, in active rheumatoid arthritis despite methotrexate therapy: the GO-FORWARD Study. Ann Rheum Dis. 2009;68(6):789-96.

13. Emery P, Breedveld F, van der Heijde D, et al. Two-year clinical and radiographic results with combination etanercept-methotrexate therapy versus monotherapy in early rheumatoid arthritis: a two-year, double-blind, randomized study. Arthritis Rheum. 2010;62(3):674-82.

14. Goekoop-Ruiterman YP, de Vries-Bouwstra JK, Allaart CF, et al. Clinical and radiographic outcomes of four different treatment strategies in patients with early rheumatoid arthritis (the BeSt study): a randomized, controlled trial. Arthritis Rheum. 2005;52(11):3381-90.

15. Choy E. Understanding the dynamics: pathways involved in the pathogenesis of rheumatoid arthritis. Rheumatology (Oxford). 2012;51(Suppl 5):v3-11.

16. Enbrel (etanercept) solution for subcutaneous use. Immunex Corporation. 2011. Available at: http://www.accessdata.fda.gov/drugsatfda docs/label/2012/103795s5507lbl.pdf. Accessed October 18, 2016.

17. Orencia (abatacept) for injection for intravenous use injection, for subcutaneous use. Bristol-Myers Squibb. Revised December 2011. Available at: http://www.accessdata.fda.gov/drugsatfda_docs/label/2012/125118s0138lbl. pdf. Accessed October 18, 2016.

18. HUMIRA (adalimumab) injection, for subcutaneous use. Abbott Laboratories. Revised September 2012. Available at: http://www.accessdata.fda.gov/drugsatfda_docs/label/2012/125057s232lbl.pdf. Accessed October 18, 2016.
19. Moots RJ, Haraoui B, Matucci-Cerinic M, et al. Differences in biologic dose-escalation, non-biologic and steroid intensification among three anti-TNF agents: evidence from clinical practice. Clin Exp Rheumatol. 2011;29(1):26-34.

20. Moots RJ, Mays R, Stephens J, Tarallo M. Burden of dose escalation with tumour necrosis factor inhibitors in rheumatoid arthritis: a systematic review of frequency and costs. Clin Exp Rheumatol. 2015;33(5):737-45.

21. Bonafede MM, Gandra SR, Fox KM, Wilson KL. Tumor necrosis factor blocker dose escalation among biologic naive rheumatoid arthritis patients in commercial managed-care plans in the 2 years following therapy initiation. J Med Econ. 2012;15(4):635-43.

22. Yazdany J, Dudley RA, Chen R, Lin GA, Tseng CW. Coverage for highcost specialty drugs for rheumatoid arthritis in Medicare Part D. Arthritis Rheumatol. 2015;67(6):1474-80.

23. Muller R, Kallikorm R, Polluste K, Lember M. Compliance with treatment of rheumatoid arthritis. Rheumatol Int. 2012;32(10):3131-35.

24. XELJANZ (tofacitinib) tablets for oral administration. Pfizer Labs. Revised November 2012. Available at: http://www.accessdata.fda.gov/drugsatfda_docs/label/2012/203214s000lbl.pdf. Accessed October 18, 2016

25. Quan H, Li B, Couris CM, et al. Updating and validating the Charlson comorbidity index and score for risk adjustment in hospital discharge abstracts using data from 6 countries. Am J Epidemiol. 2011;173(6):676-82.

26. Ting G, Schneeweiss S, Scranton R, et al. Development of a health care utilisation data-based index for rheumatoid arthritis severity: a preliminary study. Arthritis Res Ther. 2008;10(4):R95.

27. Blume SW, Fox KM, Joseph G, Chuang CC, Thomas J, Gandra SR. Tumor necrosis factor-blocker dose escalation in rheumatoid arthritis patients in a pharmacy benefit management setting. Adv Ther. 2013;30(5):517-27.

28. Ogale S, Hitraya E, Henk HJ. Patterns of biologic agent utilization among patients with rheumatoid arthritis: a retrospective cohort study. BMC Musculoskelet Disord. 2011;12:204.

29. Bonafede M, Johnson BH, Fox KM, Watson C, Gandra SR. Treatment patterns with etanercept and adalimumab for psoriatic diseases in a realworld setting. J Dermatolog Treat. 2013;24(5):369-73.

30. Nau DP. Proportion of days covered (PDC) as a preferred method of measuring medication adherence. Pharmacy Quality Alliance. 2012. Available at: http://www.pqaalliance.org/images/uploads/files/PQA\%20 PDC\%20vs\%20\%20MPR.pdf. Accessed October 18, 2016.

31. Oladapo A, Barner JC, Lawson KA, et al. Medication effectiveness with the use of tumor necrosis factor inhibitors among Texas Medicaid patients diagnosed with rheumatoid arthritis. J Manag Care Spec Pharm. 2014;20(7):657-67. Available at: http://www.jmcp.org/doi/10.18553/ jmcp.2014.20.7.657.

32. Curtis JR, Chastek B, Becker L, et al. Cost and effectiveness of biologics for rheumatoid arthritis in a commercially insured population. J Manag Care Spec Pharm. 2015;21(4):318-29. Available at: http://www.jmcp.org/ doi/10.18553/jmcp.2015.21.4.318.

33. Hochberg Y. A sharper Bonferroni procedure for multiple tests of significance. Biometrika. 1988;75(4):800-02. 
34. Vieira MC, Wallenstein G, Bradley JD, et al. Tofacitinib versus biologic treatments in patients with active rheumatoid arthritis who have had an indequate response to tumour necrosis factor inhibitors: a network metaanalysis. Ann Rheum Dis. 2013;72(S3):619.

35. Desai RJ, Solomon DH, Weinblatt ME, Shadick N, Kim SC. An external validation study reporting poor correlation between the claims-based index for rheumatoid arthritis severity and the disease activity score. Arthritis Res Ther. 2015;17(1):83.

36. Kavanaugh A, Reed GW, Saunders KC, et al. Clinical characteristics of RA patients newly prescribed tofacitinib citrate (tofacitinib) in the United States after Food and Drug Administration approval: results from the Corrona US Rheumatoid Arthritis Registry [Abstract 1537]. Arthritis Rheumatol. 2014;66(10 Suppl):S677.

37. Dougados M, Kissel K, Sheeran T et al. Adding tocilizumab or switching to tocilizumab monotherapy in methotrexate inadequate responders: 24-week symptomatic and structural results of a 2-year randomised controlled strategy trial in rheumatoid arthritis (ACT-RAY). Ann Rheum Dis. 2013;72(1):43-50

38. Weinblatt ME, Kremer J, Cush J, et al. Tocilizumab as monotherapy or in combination with nonbiologic disease-modifying antirheumatic drugs: twenty-four-week results of an open-label, clinical practice study. Arthritis Care Res (Hoboken). 2013;65(3):362-71.

39. Bykerk VP, Östör AJ, Alvaro-Gracia J, et al. Comparison of tocilizumab as monotherapy or with add-on disease-modifying antirheumatic drugs in patients with rheumatoid arthritis and inadequate responses to previous treatments: an open-label study close to clinical practice. Clin Rheumatol. 2015;34(3):563-71

40. Burmester GR, Rigby WF, van Vollenhoven RF, et al. Tocilizumab in early progressive rheumatoid arthritis: FUNCTION, a randomised controlled trial. Ann Rheum Dis. 2016;75(6):1081-91.

41. Gabay C, Riek M, Hetland ML, et al. Effectiveness of tocilizumab with and without synthetic disease-modifying antirheumatic drugs in rheumatoid arthritis: results from a European collaborative study. Ann Rheum Dis. 2016;75(7):1336-42.

42. Johnston SS, McMorrow D, Farr AM, Juneau P, Ogale S. Comparison of biologic disease-modifying antirheumatic drug therapy persistence between biologics among rheumatoid arthritis patients switching from another biologic. Rheumatol Ther. 2015;2(1):59-71.

43. Yeaw J, Benner JS, Walt JG, Sian S, Smith DB. Comparing adherence and persistence across 6 chronic medication classes. J Manag Care Pharm. 2009;15(9):728-40. Available at: http://www.jmcp.org/doi/10.18553/ jmcp. 2009.15.9.728.

44. Li P, Blum MA, von Feldt J, Hennessy S, Doshi JA. Adherence, discontinuation, and switching of biologic therapies in Medicaid enrollees with rheumatoid arthritis. Value Health. 2010;13(6):805-12.

45. Curkendall S, Patel V, Gleeson M, Campbell RS, Zagari M, Dubois R. Compliance with biologic therapies for rheumatoid arthritis: do patient outof-pocket payments matter? Arthritis Rheum. 2008;59(10):1519-26.

46. Contreras-Yanez I, Ponce De Leon S, Cabiedes J, Rull-Gabayet M, Pascual-Ramos V. Inadequate therapy behavior is associated to disease flares in patients with rheumatoid arthritis who have achieved remission with disease-modifying antirheumatic drugs. Am J Med Sci. 2010;340(4):282-90.
47. Saverno K, Louder A, Singh A, et al. Understanding patient preferences associated with the use of therapies for rheumatoid arthritis: results of a conjoint analysis [Abstract 2395]. Arthritis Rheum. 2014;66(10 Suppl):S1043. 48. Williams EL, Edwards CJ. Patient preferences in choosing anti-TNF therapies-R1. Rheumatology (Oxford). 2006;45(12):1575-76.

49. Souto A, Maneiro JR, Gómez-Reino JJ. Rate of discontinuation and drug survival of biologic therapies in rheumatoid arthritis: a systematic review and meta-analysis of drug registries and health care databases. Rheumatology (Oxford). 2016;55(3):523-34.

50. Neovius M, Arkema EV, Olsson H, et al. Drug survival on TNF inhibitors in patients with rheumatoid arthritis comparison of adalimumab, etanercept and infliximab. Ann Rheum Dis. 2015;74(2):354-60.

51. Frazier-Mironer A, Dougados M, Mariette X, et al. Retention rates of adalimumab, etanercept and infliximab as first and second-line biotherapy in patients with rheumatoid arthritis in daily practice. Joint Bone Spine. 2014;81(4):352-59.

52. Fidder HH, Singendonk MM, van der Have M, Oldenburg B, van Oijen MG. Low rates of adherence for tumor necrosis factor- $\alpha$ inhibitors in Crohn's disease and rheumatoid arthritis: results of a systematic review. World J Gastroenterol. 2013;19(27):4344-50.

53. Cannon GW, DuVall SL, Haroldsen CL, et al. Persistence and dose escalation of tumor necrosis factor inhibitors in U.S. veterans with rheumatoid arthritis. J Rheumatol. 2014;41(10):1935-43.

54. Fisher A, Bassett K, Wright JM, Brookhart MA, Freeman H, Dormuth CR. Comparative persistence of the TNF antagonists in rheumatoid arthritis-a population-based cohort study. PLoS One. 2014;9(8):e105193.

55. Tang B, Rahman M, Waters HC, Callegari P. Treatment persistence with adalimumab, etanercept, or infliximab in combination with methotrexate and the effects on health care costs in patients with rheumatoid arthritis. Clin Ther. 2008;30(7):1375-84.

56. Curtis JR, Zhang J, Xie F, et al. Use of oral and subcutaneous methotrexate in rheumatoid arthritis patients in the United States. Arthritis Care Res (Hoboken). 2014;66(11):1604-11.

57. Bergvall N, Petrilla AA, Karkare SU, et al. Persistence with and adherence to fingolimod compared with other disease-modifying therapies for the treatment of multiple sclerosis: a retrospective U.S. claims database analysis. J Med Econ. 2014;17(10):696-707.

58. He A, Spelman T, Jokubaitis V, et al. Comparison of switch to fingolimod or interferon beta/glatiramer acetate in active multiple sclerosis. JAMA Neurol. 2015;72(4):405-13.

59. Warrender-Sparkes M, Spelman T, Izquierdo G, et al. The effect of oral immunomodulatory therapy on treatment uptake and persistence in multiple sclerosis. Mult Scler. 2016;22(4):520-32.

60. Harnett J, Wiederkehr D, Gerber R, Gruben D, Bourret J, Koenig A. Primary non-adherence, associated clinical outcomes and healthcare resource use among patients with rheumatoid arthritis prescribed treatment with injectable biologic disease-modifying antirheumatic drugs. J Manag Care Spec Pharm. 2016;22(3):209-18. Available at: http://www.jmcp.org/doi/ full/10.18553/jmcp.2016.22.3.209. 
Evaluation of Real-World Experience with Tofacitinib Compared with Adalimumab, Etanercept, and Abatacept in RA Patients with 1 Previous Biologic DMARD: Data from a U.S. Administrative Claims Database

61. Harnett J, Wiederkehr D, Gerber R, Gruben D, Koenig A, Bourret J. Real-world evaluation of TNF-inhibitor utilization in rheumatoid arthritis. J Med Econ. 2016;19(2):91-102.

62. Borah BJ, Huang X, Zarotsky V, Globe D. Trends in RA patients' adherence to subcutaneous anti-TNF therapies and costs. Curr Med Res Opin. 2009;25(6):1365-77.

63. Tkacz J, Ellis L, Bolge SC, Meyer R, Brady BL, Ruetsch C. Utilization and adherence patterns of subcutaneously administered anti-tumor necrosis factor treatment among rheumatoid arthritis patients. Clin Ther. 2014;36(5):737-47.
64. Chu LH, Kawatkar AA, Gabriel SE. Medication adherence and attrition to biologic treatment in rheumatoid arthritis patients. Clin Ther. 2015;37(3):660-66.

65. Campagna EJ, Muser E, Parks J, Morrato EH. Methodological considerations in estimating adherence and persistence for a long-acting injectable medication. J Manag Care Spec Pharm. 2014;20(7):756-66. Available at: http://www.jmcp.org/doi/10.18553/jmcp.2014.20.7.756. 
Evaluation of Real-World Experience with Tofacitinib Compared with Adalimumab, Etanercept, and Abatacept in RA Patients with 1 Previous Biologic DMARD: Data from a U.S. Administrative Claims Database

APPENDIX Independent Variables Used in Adjusted Analyses

Independent Variable

Age at index

Region of the United States

Sex

Insurance type of index claim

Length of pre-index enrollment

Quan-Charlson index during l-year pre-index period

Pre-index CIRAS

Number of days between first RA diagnosis during baseline and the index date

csDMARD prescription count during 1 year pre-index period

Index medication

Use of $\geq 1$ of main csDMARDs during 90-day post-index period

Rheumatologist visit count during l-year pre-index period

Unique number of opioid prescriptions (by NDC number) during l-year pre-index period

TNFi use during whole database pre-index period

bDMARD use during l-year pre-index period

Corresponding costs during 1-year pre-index period

Categories for Categorical Independent Variables

$A B A=$ abatacept $A D A=$ adalimumab; $b D M A R D=$ biologic disease-modifying antirheumatic drug; CIRAS = claims-based index of RA severity; csDMARD = conventional

synthetic disease-modifying antirheumatic drug; ETN=etanercept; NDC=National Drug Code; RA=rheumatoid arthritis; TNFi=tumor necrosis factor inhibitor. 